\title{
Serum miRNA-122 is an Independent Biomarker of Survival in Patients with Primary Sclerosing Cholangitis
}

\author{
Kilian Friedrich ${ }^{1}$, Carina Baumann ${ }^{1}$, Andreas Wannhoff ${ }^{1}$, Christian Rupp ${ }^{1}$, Arianeb Mehrabi ${ }^{2}$, Karl Heinz Weiss ${ }^{1}$, Daniel \\ N. Gotthardt ${ }^{1}$
}

1) University Hospital of

Heidelberg, Department IV, Im Neuenheimer Feld 410, Heidelberg,

2) Department of Surgery, University Hospital of Heidelberg, Im Neuenheimer Feld 110, Heidelberg Germany

Address for correspondence: Kilian Friedrich Im Neuenheimer Feld 410, 69120 Heidelberg, Germany info@gastropraxis-friedrich.de

Received: 12.02 .2018

Accepted: 28.05.2018

\begin{abstract}
Background \& Aims: The disease course of primary sclerosing cholangitis (PSC) is variable and difficult to predict. MicroRNA-122 (miR-122) is associated with various liver diseases. We investigated the value of miR-122 as a biomarker for the disease course of PSC.

Methods: We determined serum miR-122 levels in a long-term, prospective cohort of 114 PSC patients and a second validation cohort.

Results: Based on miR-122 levels, PSC patients were assigned to low or high level miR-122 groups. KaplanMeier analysis showed significantly impaired actuarial transplant-free survival for PSC patients in the low miR-122 group (mean: $46.1 \pm 4.1$ months; $95 \%$ confidence intervals [CI]: 38.1-54.2) compared to the high miR122 group (mean: $95.2 \pm 7.9$ months; $95 \%$ CI: 79.5-110.8; $\mathrm{p}=0.034$ ). Using a multivariate Cox's proportional hazards model approach, Mayo-Risk score (odds ratio [OR]: 1.47; 95\% CI: 1.13-1.92; $\mathrm{p}=0.004$ ), the presence of dominant strictures (OR: 2.62; 95\% CI: 1.00-5.55; $\mathrm{p}=0.004$ ), and serum miR-122 levels (OR: 1.19; 95\% CI: 1.00-1.43; $\mathrm{p}=0.045)$ were independent risk factors associated with reduced actuarial transplant-free survival. We were able to confirm this finding in a second, independent cohort of PSC patients (low miR-122 group: mean survival: $13.1 \pm 5.2$ months; $95 \%$ CI: 2.8-23.4; high miR-122 group: mean: $28.62 \pm 4.2$ months; $95 \%$ CI: 20.3-37.0; $\mathrm{p}=0.018$ ).

Conclusions: We identified miR-122 as a novel, independent prognostic biomarker associated with improved survival in PSC patients. It is unknown whether exogenous miR-122 might influence the disease course of PSC patients.
\end{abstract}

Key words: miR-122 - primary sclerosing cholangitis - actuarial survival free of liver transplantation biomarker.

Abbreviations: AP: alkaline phosphatase; CT: cycle threshold; DS: dominant stenosis; ERC: endoscopic retrograde cholangiography; IBD: inflammatory bowel disease; miR-122: microRNA-122; PSC: primary sclerosing cholangitis; RT-qPCR: quantitative real-time reverse transcription polymerase chain reaction.

\section{INTRODUCTION}

Primary sclerosing cholangitis (PSC) is a chronic inflammatory disease characterized by the destruction and strictures of intrahepatic and extrahepatic biliary ducts [1-3]. This progressive disorder primarily affects young males and ultimately leads to biliary cirrhosis, portal hypertension, and hepatic failure $[4,5]$. Because disease progression is characterized by individual variation [6,7], the identification of prognostic factors for PSC patients is of utmost importance.

MicroRNAs (miRNAs) are small endogenous RNAs that post-transcriptionally control gene expression. They are expressed in multiple tissues and are important in multiple regulatory mechanisms including cell differentiation, proliferation, apoptosis, and tumorigenesis [8-10]. miR-122 is primarily expressed in the liver and constitutes $70 \%$ of the total miRNA population [11]. Compared to its expression in healthy liver tissue, miR-122 expression is markedly reduced in hepatocellular carcinoma [12] and correlates with a poor patient prognosis [13]. Furthermore, miR-122 has been shown to regulate fat and cholesterol metabolism [14]. Recently, miR122 has also been associated with disease activity in chronic hepatitis $C$ and drug-induced liver injury $[15,16]$. Because 
miR-122 alterations occur prior to changes in aminotransferase activity, it has been proposed as a predictive blood marker for viral-, alcohol-, and chemical-induced liver injury [17].

Interestingly, miR-122 deficiency shares certain commonalities with PSC as both demonstrate inflammatory recruitment abnormalities [18], cholestatic hepatobiliary disease [19], and ultimately lead to the development of liver fibrosis $[19,20]$. Additionally, key enzymes involved in bile acid homeostasis are regulated by miR-122 [21]. Therefore, the aim of this study was to investigate the predictive value of serum miR-122 levels in a prospective, long-term cohort of PSC patients.

\section{METHODS}

\section{Study design}

The prospective study began on May 1, 1987. Patients were included in this study until 2011 and clinical follow up was conducted until 2013. After obtaining written consent, serum samples were collected from 114 patients ultimately included in the study cohort (Table I). The study was approved by the Ethics Committee of the University of Heidelberg and informed consent to participate in the study was obtained from each subject in compliance with this committee. The study was carried out in accordance with the Declaration of Helsinki.

As previously described $[22,23]$, selection criteria for enrolling patients with PSC in the prospective study included typical endoscopic retrograde cholangiographic (ERC) findings, serum alkaline phosphatase (AP) activity of at least twice the normal range, negative antimitochondrial antibody titers, and a liver biopsy compatible with the diagnosis of PSC. Exclusion criteria included decompensated liver

Table I. Patients characteristics

\begin{tabular}{lcc}
\hline & $\begin{array}{c}\text { Prospective } \\
\text { PSC cohort }\end{array}$ & $\begin{array}{c}\text { Second PSC } \\
\text { cohort }\end{array}$ \\
\hline Patients (n) & 114 & 30 \\
Gender, male/female, n (\%) & $80 / 34$ & $22 / 8$ \\
& $(70.1 / 29.8)$ & $(73.3 / 26.6)$ \\
Age at diagnosis [years], mean, SD & $34.9 \pm 13.3$ & $29.2 \pm 10.7$ \\
IBD, n (\%) & $79(69.2)$ & $22(73.3)$ \\
Dominant stenosis, n (\%) & $73(64.0)$ & $22(73.3)$ \\
Liver transplantation, n (\%) & $20(17.5)$ & $9(30.0)$ \\
Death, n (\%) & $7(6.1)$ & $1(3.3)$ \\
CCA, n (\%) & $4(3.5)$ & 0 \\
Mayo risk score, mean, SD & $0.070 \pm 1.2$ & $0.26 \pm 1.1$ \\
ALT (U/l), mean, SD & $105.3 \pm 99.9$ & $91.7 \pm 78.1$ \\
AST (U/l), mean, SD & $68.1 \pm 55.2$ & $75.1 \pm 52.5$ \\
GGT (U/l), mean, SD & $291.4 \pm 314.2$ & $262.1 \pm 308.8$ \\
ALP (U/l), mean, SD & $285.1 \pm 230.4$ & $310.1 \pm 196.6$ \\
Albumin (g/dl), mean, SD & $4.2 \pm 7.86$ & $4.8 \pm 5.52$ \\
Bilirubin (mg/dl), mean, SD & $1.69 \pm 2.12$ & $2.42 \pm 2.43$ \\
INR, mean, SD & $1.0 \pm 0.12$ & $1.06 \pm 0.07$ \\
CRP (mg/l), mean, SD & $11.8 \pm 20.2$ & $13.9 \pm 23.0$ \\
Leucocytes (/nl), mean, SD & $7.5 \pm 3.5$ & $7.3 \pm 2.4$ \\
\hline CCA: cholangiocarcinoma CRP: & \\
\hline
\end{tabular}

CCA: cholangiocarcinoma; CRP: $\mathrm{C}$ reactive protein. cirrhosis, impending liver transplantation, a diagnosis of cholangiocellular carcinoma occurring within three months after presentation, and a history of neoplastic disease and/or hepatic comorbidity.

All subjects underwent a yearly physical examination, biochemical blood tests, and abdominal ultrasound. Additionally, all patients received treatment with ursodeoxycholic acid. The biliary system was evaluated with ERC in all patients upon entry into the study. In cases where dominant stenosis (DS) was present, ERC was repeated yearly. If there was no narrowing of the common bile duct at entry into the study, ERC was performed every two years until 1995. Since all patients with DS showed an increase in liver enzymes, starting in 1995, repeat ERC in patients without DS was only performed when AP or gamma-glutamyltransferase (GGT) increased $\geq 20 \%$. A DS was defined as a common bile duct stenosis of $<1.5 \mathrm{~mm}$ diameter or a hepatic duct stenosis of $<$ $1.0 \mathrm{~mm}$ within $2 \mathrm{~cm}$ of the bifurcation. In patients with total or subtotal stenosis of the common bile duct and biochemical evidence of cholestasis, rigid dilatation followed by balloon dilatation of the stenosis was performed after endoscopic sphincterotomy of the papilla. In patients with dominant stenosis of the common bile duct, ERC was repeated at yearly intervals. Serum samples of prospective study cohort patients were obtained either at inclusion to the study or if not available at a later time point. For miRNA analysis, the serum samples obtained at the earliest time point after study inclusion were used.

Patients in the second PSC study cohort were transferred to our center for evaluation of liver function and liver transplantation. Because these patients were consequently enrolled at Eurotransplant for liver transplantation, they were not included in the prospective study cohort.

\section{Blood sampling and miRNA determination}

Blood serum samples were obtained between 8 to 9 a.m. in the fasted state. Blood samples were then immediately frozen at minus 80 degrees Celsius. Human RNA was extracted using the miRNeasy serum/blood plasma kit (Qiagen, Venlo, Netherlands), which utilizes phenol and chloroform, and plasma spiked with Caenorhabditis elegans miR-39 mimic (Qiagen, Venlo, Netherlands) as a positive control. Human miR-122 expression was measured by quantitative real-time reverse transcription polymerase chain reaction (RT-qPCR) in a fluorescent temperature cycler using the TaqMan assay. Fluorescence was detected on an ABI PRISM 7000 sequence detector (Applied Biosystems, Darmstadt, Germany). Applied Biosystems’ TaqMan ${ }^{\oplus}$ miRNA Reverse Transcription Kit (Life Technologies GmbH, Darmstadt, Germany) was used together with $\operatorname{TaqMan}^{\circledast}$ miRNA Assay Primer for hsa-miR-122 to quantify the miRNA level. Since there are not established cutoff values for miRNA-122 available, we decided to use the mean miRNA cycle threshold (CT)-levels (28.5) measured by RTqPCR for dichotomizing to miRNA-122 low- and high groups.

\section{Statistical analysis}

The Student's $t$-test was performed to compare the means of the two groups for continuous numerical data. Spearman's rho was used as the nonparametric measure of statistical dependence 
between two variables. The actuarial liver transplant-free survival rate was estimated using the Kaplan-Meier method. Differences between the actuarial liver transplant-free survival rate estimates were analyzed using the log-rank test. The following variables were selected for univariate analysis based on the results of previous studies: Mayo-Risk score, inflammatory bowel disease (IBD), the presence of dominant strictures, and serum miR-122 levels. A p-value of $<0.1$ in univariate analysis was defined for variables to be included in a Cox's proportional hazards model, using a stepwise procedure with a threshold of $\alpha=0.05$.

\section{RESULTS}

Patient characteristics in the prospective study cohort

The prospective study cohort consisted of 114 PSC patients of whom 34 were female $(29.8 \%)$ and 80 were male $(70.1 \%)$. Mean age at PSC diagnosis was 34.9 years $( \pm 13.3)$. Twenty patients $(17.5 \%)$ received liver transplantation while 7 patients (6.1\%) died due to end-stage liver disease during follow-up. Mean follow up time from time of serum sampling was 79.5 \pm 6.0 months (95\% CI: 67.6-91.4).

Distribution of serum miR-122 levels in PSC patients

Time from blood sampling until miR-122 analysis after sample thawing was $78.9 \pm 26.6$ months. Figure 1 shows the distribution pattern of serum miR-122 levels. Based on the mean miRNA cycle threshold (CT)-levels (28.5) measured by RT-qPCR we dichotomized the patients into a low miR-122 level group (CT-value $>28.5$ ) and a high miR-122 level group (CT-value $\leq 28.5$ ). There was no statistical difference regarding liver function parameters, liver transaminases, and cholestatic or inflammatory markers between the two groups at the beginning of the study (Table II). Notably, liver transplantation occurred significantly more often in the low miR-122 group $(28.3 \%$ vs. $5.6 \%, \mathrm{p}=0.001)$.

Serum miR-122 levels as a marker for actuarial liver transplant-free survival in PSC patients

Actuarial liver transplant-free survival determined by Kaplan-Meier analysis was significantly impaired for PSC

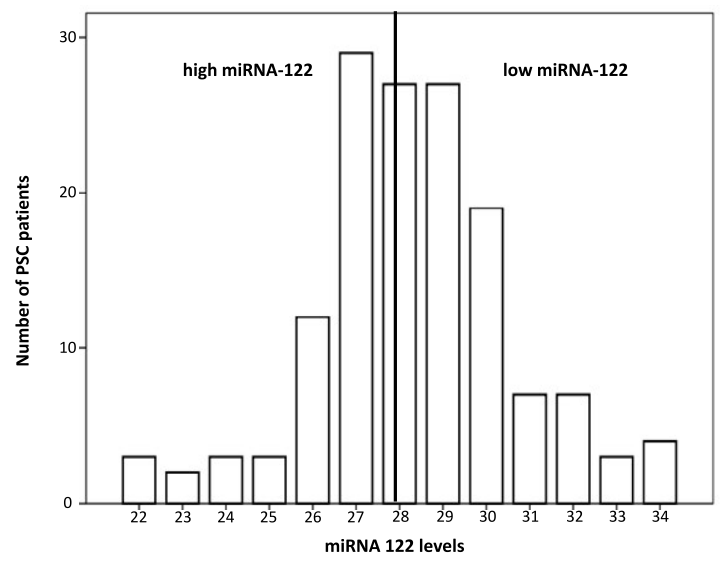

Fig. 1. Distribution of serum miR-122 levels. The cut-off value for the patient cohorts is marked in red. Patients were assigned to the low miRNA-122 group (CT-value $>28.5$ ) or the high miRNA-122 group (CT-value $\leq 28.5)$ based on the mean miRNA CT-levels (28.5) as measured by RT-qPCR.
Table II. Correlation between miR-122 serum levels and laboratory parameters.

\begin{tabular}{|c|c|c|c|}
\hline Parameter & $\begin{array}{l}\text { Low miR-122 } \\
\text { group }\end{array}$ & $\begin{array}{l}\text { High miR-122 } \\
\text { group }\end{array}$ & p-value \\
\hline Patients (n) & 60 & 54 & n.a. \\
\hline Gender, male/female, n (\%) & $45 / 15$ & $35 / 19$ & 0.235 \\
\hline $\begin{array}{l}\text { Age at diagnosis [years], } \\
\text { mean, SD }\end{array}$ & $34.5 \pm 12.8$ & $35.3 \pm 13.4$ & 0.725 \\
\hline IBD, n (\%) & $44(73.3)$ & $35(64.8)$ & 0.325 \\
\hline Dominant stenosis, n (\%) & $38(63.3)$ & $39(72.2)$ & 0.311 \\
\hline Liver transplantation, $\mathrm{n}(\%)$ & $17(28.3)$ & $3(5.6)$ & 0.001 \\
\hline Death, n (\%) & $4(6.6)$ & $3(5.5)$ & 0.805 \\
\hline CCA, n (\%) & $3(5.0)$ & $1(1.8)$ & 0.345 \\
\hline Mayo-risk score, mean, SD & $0.014 \pm 1.22$ & $0.147 \pm 1.25$ & 0.506 \\
\hline $\operatorname{ALT}(\mathrm{U} / \mathrm{l})$, mean, SD & $88.8 \pm 96.1$ & $124.6 \pm 101.8$ & 0.066 \\
\hline AST (U/1), mean, SD & $59.7 \pm 47.9$ & $77.6 \pm 61.7$ & 0.096 \\
\hline GGT (U/l), mean, SD & $243.6 \pm 316.7$ & $284.1 \pm 183.0$ & 0.073 \\
\hline $\operatorname{ALP}(\mathrm{U} / \mathrm{l})$, mean, $\mathrm{SD}$ & $293.7 \pm 267.8$ & $284.1 \pm 183.0$ & 0.833 \\
\hline Albumin (g/dl), mean, SD & $4.1 \pm 8.8$ & $4.3 \pm 6.7$ & 0.416 \\
\hline Bilirubin (mg/dl), mean, SD & $1.9 \pm 2.5$ & $1.4 \pm 1.6$ & 0.196 \\
\hline INR, mean, SD & $1.09 \pm .13$ & $1.04 \pm .11$ & 0.223 \\
\hline CRP (mg/l), mean, SD & $13.6 \pm 20.7$ & $9.0 \pm 20.1$ & 0.354 \\
\hline Leucocytes $(/ \mathrm{nl})$, mean, SD & $7.8 \pm 4.0$ & $6.6 \pm 1.9$ & 0.186 \\
\hline
\end{tabular}

patients in the low miR-122 group (mean: $46.1 \pm 4.1$ months; 95\% CI: 38.1-54.2) compared to the high miR-122 group (mean: $95.2 \pm 7.9$ months; 95\% CI: 79.5-110.8; $\mathrm{p}=0.034$ ) (Fig. 2 ). While 24 patients $(40.0 \%)$ with low miR-122 levels died or underwent liver transplantation, death or transplantation occurred in only 9 patients (16.6\%) in the high miR-122 group.

Multivariate analysis for actuarial transplant-free survival Mayo-Risk score, IBD, the presence of dominant strictures, and serum miR-122 levels were subjected to Cox

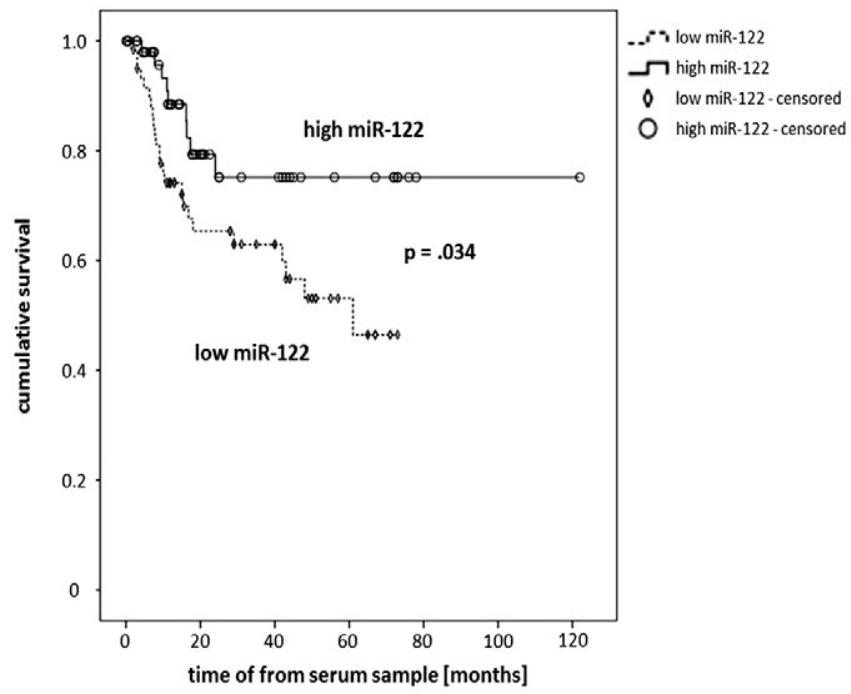

Fig. 2. Actuarial liver transplantat-free survival based on serum miR122 levels. Kaplan-Meier estimate of all patients in the prospective study cohort $(n=114)$ showed markedly impaired actuarial transplantfree survival in the presence of a dominant stenosis (DS) $(\mathrm{p}=0.034)$. 
univariate analysis. All variables were associated with actuarial transplant-free survival below the set p-value of 0.1 (Table III) and therefore subjected to further multivariate analysis. In multivariate analysis, Mayo-Risk score, the presence of dominant strictures, and serum miR-122 levels remained independent risk factors associated with reduced actuarial transplant-free survival.

Table III. Features associated with death/Ltx in PSC patients on liver transplant list according to Cox's proportional hazards model.

\begin{tabular}{lcc}
\hline & $\begin{array}{c}\text { Cox univariate analysis } \\
\text { OR }[95 \% \mathrm{CI}]\end{array}$ & $\begin{array}{c}\text { Cox multivariate analysis } \\
\text { OR [95\% CI] }\end{array}$ \\
\hline miRNA-122 & $1.27[1.04-1.39]$ & $1.19[1.00-1.43]$ \\
Mayo-Risk & $\mathrm{p}=0.009$ & $\mathrm{p}=0.045$ \\
score & $1.35[1.06-1.73]$ & $1.47[1.13-1.92]$ \\
IBD & $\mathrm{p}=0.015$ & $\mathrm{p}=0.004$ \\
& $2.36[.966-5.77]$ & $2.54[.94-6.82]$ \\
Dominant & $\mathrm{p}=0.059$ & $\mathrm{p}=0.064$ \\
stricture & $2.00[.903-4.44]$ & $2.62[1.00-5.55]$ \\
\hline & $\mathrm{p}=0.087$ & $\mathrm{p}=0.049$
\end{tabular}

Subjection of AP and miR-122 levels to multivariate analysis showed that both factors were significantly associated with actuarial transplant-free survival (AP: 1.0 [1.001-1.003], $\mathrm{p}=0.001 ; \mathrm{miR}-122: 1.19$ [1.02-1.40], $\mathrm{p}=0.027)$.

Patient characteristics in a second cohort of PSC patients

The second cohort consisted of 22 male and 8 female PSC patients (73.3\% vs. $26.6 \%)$. Mean age of PSC diagnosis was at 29.2 years $( \pm 10.7)$. From the time of serum sampling, mean follow-up time in this cohort was 11.3 months $( \pm 10.6)$. At time of serum sampling, Mayo-Risk score was $0.26 \pm 1.1$. Mean follow-up from the time of serum sampling was 27.6 months ( \pm 24.2). During the course of the PSC disease, 9 patients received liver transplantation while 1 patient died during follow-up due to end-stage liver disease. Mean follow up time from time of serum sampling was $23.3 \pm 3.2$ months (95\% CI: 16.9-31.5).

\section{Serum miR-122 levels in the second PSC cohort}

Time from blood sampling until miR-122 analysis after sample thawing was $48.8 \pm 11.7$ months. In the second PSC cohort, serum miR-122 levels did not correlate with Mayo-Risk score $(\mathrm{p}=0.115)$, serum bilirubin $(\mathrm{p}=0.066)$, AST $(\mathrm{p}=0.414)$, $\operatorname{ALT}(\mathrm{p}=0.311)$, GGT $(\mathrm{p}=0.461), \operatorname{AP}(\mathrm{p}=0.073)$, albumin ( $=0.842)$ and $\operatorname{INR}(\mathrm{p}=0.064)$. Patients were dichotomized into a low miR-122 level group (CT-value $>28.5$ ) and a high miR-122 level group (CT-value $\leq 28.5$ ) as done in the prospective PSC cohort. Between these two cohorts, there were no statistical differences regarding clinical and serological parameters at the time of serum sampling (Supplemental Table I).

Serum miR-122 levels and actuarial liver transplant-free survival in the second cohort of PSC patients

Actuarial transplant-free survival was significantly impaired for patients with low miR-122 levels (mean: $13.1 \pm$ 5.2 months; 95\% CI: 2.8-23.4) compared with that in those with high miR-122 levels (mean: $28.6 \pm 4.2$ months; $95 \%$ CI: $20.3-37.0 ; \mathrm{p}=0.018)$. While 6 patients $(40.0 \%)$ with low miR-122 levels died or received liver transplantation, only 4 patients (19.0\%) with high miR-122 levels died or received liver transplantation.

\section{DISCUSSION}

Changes in hepatic and serum miR-122 levels have been associated with a variety of hepatic disorders such as alcoholic, viral, fibrotic, and fatty liver disease [24-28]. As miR-122 is stable and can be reliably detected in human sera [29], it has been proposed as a biomarker for hepatic disorders [30, 31]. Due to the highly unpredictable disease course of PSC patients [1], identification of reliable biomarkers is urgently needed, particularly since the only available cure is liver transplantation which may not be available to all patients.

In this long-term, prospective study of PSC patients, we showed that a reduction of serum miR-122 levels is associated with impaired actuarial liver transplant-free survival, and demonstrated that miR-122 is an independent predictor of survival in PSC patients as shown by multivariate analysis (Table III). We were able to confirm this finding in a second, independent cohort of PSC patients. Reduction in alkaline phosphatase (AP) has recently been shown to be associated with longer survival in PSC [32]. Again, when subjected to multivariate analysis, both AP and miR-122 remained as independent predictors of survival. These findings are in agreement with a prior study showing that serum microRNA-122 predicts survival in patients with liver cirrhosis [33]. This association, however, was mainly attributed to endstage liver disease, as Waidman et al. [33] reported a highly significant correlation of liver function impairment and miR122 levels. In contrast to the study of Waidman et al. [33], patients with end-stage liver disease were not included in the prospective study cohort based on study protocol. Since miR122 levels did not correlate with Mayo-Risk score (Pearson correlation coefficient: $-0.007 ; \mathrm{p}=0.940$ ) at the beginning of the study, this indicates the predictive value of miR-122 in PSC patients.

Interestingly, a deficiency of miR-122 shares common features of biliary damage. Six-month-old miR-122 knockout mice exhibit significantly increased serum AP and GGT levels, consistent with hepatobiliary disease [19]. Furthermore, cholesterol 7a-hydroxylase (CYP7A1) and the canalicular bile salt export pump are protagonists in bile acid secretion and synthesis, both representing direct targets of human miR-122a [21]. Additionally, constitutively deficient miR-122 knockout mice show an increased recruitment of inflammatory cells such as CD11bhiGr1+, which are known to produce high levels of interleukin- 6 and tumor necrosis factor- $\alpha$ [18], ultimately resulting in liver fibrosis $[19,20]$. It is intriguing to speculate that our observed correlation of miR-122 levels and impaired survival might be attributable to the increased inflammation observed with reduced miR-122 levels.

An association of decreased miR-122 and the development of hepatocellular carcinoma has been widely reported. It has been presumed that miR-122 functions as a tumor-suppressor gene $[13,34]$. In this respect, we observed a trend towards increased cholangiocarcinoma development in the low miR122 group compared to that in the high miR-122 group (5\% vs. $1.8 \%$ respectively, Table II). However, this was not statistically 
significant. Furthermore, we did not observe a correlation between miRNA-122 levels and the presence of IBD in this study.

\section{CONCLUSION}

In this prospective, long-term PSC study, we demonstrated that serum miR-122 levels represent an independent predictor of transplant-free survival. It is unknown whether exogenous miR-122 can ameliorate the disease course of PSC patients, particularly taking into account that the therapeutic delivery of miRNA remains a major challenge. Future studies are required to validate these findings in the context of the timing of liver transplantation and as a substitute for interventional studies.

Conflicts of interest: No conflicts to declare.

Authors' contributions: K.F., W.S., D.N.G.: study concept and design; K.F., C.B., A.W. data acquisition; K.F., D.N.G., K.H.W: data analysis and interpretation; K.H.W.and A.M.: critical revision of the manuscript for important intellectual content: K.F. and D.N.G.: statistical analysis; K.F., C.R. and D.N.G.: technical, or material support.

Supplementary material: To access the supplementary material visit the online version of the J Gastrointestin Liver Dis at http://dx.doi. org/10.15403/jgld.2014.1121.272.cho

\section{REFERENCES}

1. Chapman R, Fevery J, Kalloo A, et al. Diagnosis and management of primary sclerosing cholangitis. Hepatology 2010;51:660-678. doi:10.1002/hep.23294

2. Pollheimer MJ, Halilbasic E, Fickert P, Trauner M. Pathogenesis of primary sclerosing cholangitis. Best Pract Res Clin Gastroenterol 2011;25:727-739. doi:10.1016/j.bpg.2011.10.009

3. Beuers U, Maillette de Buy Wenniger LJ, Doorenspleet M, et al. IgG4associated cholangitis. Dig Dis 2014;32:605-608. doi:10.1159/000360513

4. Chapman R, Cullen S. Etiopathogenesis of primary sclerosing cholangitis. World J Gastroenterol 2008;14:3350-3359. doi:10.3748/ wjg. 14.3350

5. Ponsioen C. Diagnosis, prognosis, and management of primary sclerosing cholangitis. Gastroenterol Hepatol (N Y) 2013;9:453-465.

6. Tischendorf JJ, Hecker H, Krüger M, Manns MP, Meier PN. Characterization, outcome, and prognosis in 273 patients with primary sclerosing cholangitis: A single center study. Am J Gastroenterol 2007;102:107-114. doi:10.1111/j.1572-0241.2006.00872.x

7. Bambha K, Kim WR, Talwalkar J, et al. Incidence, clinical spectrum, and outcomes of primary sclerosing cholangitis in a United States community. Gastroenterology 2003;125:1364-1369. doi:10.1016/j. gastro.2003.07.011

8. Wang XW, Heegaard NH, Orum H. MicroRNAs in liver disease. Gastroenterology 2012;142:1431-1443. doi:10.1053/j. gastro.2012.04.007

9. Hsu SH, Ghoshal K. MicroRNAs in Liver Health and Disease. Curr Pathobiol Rep 2013;1:53-62. doi:10.1007/s40139-012-0005-4

10. Hu J, Xu Y, Hao J, Wang S, Li C, Meng S. MiR-122 in hepatic function and liver diseases. Protein Cell 2012;3:364-371. doi:10.1007/s13238012-2036-3
11. Chang J, Nicolas E, Marks D, et al. miR-122, a mammalian liver-specific microRNA, is processed from hcr mRNA and may downregulate the high affinity cationic amino acid transporter CAT-1. RNA Biol 2004;1:106-113.

12. Kutay H, Bai S, Datta J, et al. Downregulation of miR-122 in the rodent and human hepatocellular carcinomas. J Cell Biochem 2006;99:671-678. doi: $10.1002 / j c b .20982$

13. Coulouarn C, Factor VM, Andersen JB, Durkin ME, Thorgeirsson SS. Loss of miR-122 expression in liver cancer correlates with suppression of the hepatic phenotype and gain of metastatic properties. Oncogene 2009;28:3526-3536. doi:10.1038/onc.2009.211

14. Wen J, Friedman JR. miR-122 regulates hepatic lipid metabolism and tumor suppression. J Clin Invest 2012;122:2773-2776. doi:10.1172/ JCI63966

15. Wang K, Zhang S, Marzolf B, et al. Circulating microRNAs, potential biomarkers for drug-induced liver injury. Proc Natl Acad Sci U S A 2009;106:4402-4407. doi:10.1073/pnas.0813371106

16. Bihrer V, Friedrich-Rust M, Kronenberger B, et al. Serum miR-122 as a biomarker of necroinflammation in patients with chronic hepatitis $\mathrm{C}$ virus infection. Am J Gastroenterol 2011;106:1663-1669. doi:10.1038 ajg.2011.161

17. Li ZY, Xi Y, Zhu WN, et al. Positive regulation of hepatic miR-122 expression by HNF4alpha. J Hepatol 2011;55:602-611. doi:10.1016/j. jhep.2010.12.023

18. Karlmark KR1, Weiskirchen R, Zimmermann HW, et al. Hepatic recruitment of the inflammatory Gr1+ monocyte subset upon liver injury promotes hepatic fibrosis. Hepatology 2009;50:261-274. doi:10.1002/hep.22950

19. Hsu SH, Wang B, Kota J, et al. Essential metabolic, anti-inflammatory, and anti-tumorigenic functions of miR-122 in liver. J Clin Invest 2012;122:2871-2883. doi:10.1172/JCI63539

20. Tsai WC, Hsu SD, Hsu CS, et al. MicroRNA-122 plays a critical role in liver homeostasis and hepatocarcinogenesis. J Clin Invest 2012;122:2884-2897. doi:10.1172/JCI63455

21. Marin JJ, Bujanda L, Banales JM. MicroRNAs and cholestatic liver diseases. Curr Opin Gastroenterol 2014;30:303-309. doi:10.1097/ MOG.0000000000000051

22. Gotthardt DN, Rudolph G, Klöters-Plachky P, Kulaksiz H, Stiehl A. Endoscopic dilation of dominant stenoses in primary sclerosing cholangitis: outcome after long-term treatment. Gastrointest Endosc 2010;71:527-534. doi:10.1016/j.gie.2009.10.041

23. Friedrich $\mathrm{K}$, Rupp C, Hov JR, et al. A frequent PNPLA3 variant is a sex specific disease modifier in PSC patients with bile duct stenosis. PLoS One 2013;8:e58734. doi:10.1371/journal.pone.0058734

24. Liu Y, Chen SH, Jin X, Li YM. Analysis of differentially expressed genes and microRNAs in alcoholic liver disease. Int J Mol Med 2013;31:547554. doi:10.3892/ijmm.2013.1243

25. Kamo Y, Ichikawa T, Miyaaki H, et al. Significance of miRNA-122 in chronic hepatitis $\mathrm{C}$ patients with serotype 1 on interferon therapy. Hepatol Res 2015;45:88-96. doi:10.1111/hepr.12317

26. Fukuhara T, Matsuura Y. Role of miR-122 and lipid metabolism in HCV infection. J Gastroenterol 2013;48:169-176. doi:10.1007/s00535-0120661-5

27. Miyaaki H, Ichikawa $T$, Kamo $Y$, et al. Significance of serum and hepatic microRNA-122 levels in patients with non-alcoholic fatty liver disease. Liver Int 2014;34:e302-e307. doi:10.1111/liv.12429

28. Du Y, Han X, Pu R, Xie J, Zhang Y, Cao G. Association of miRNA122-binding site polymorphism at the interleukin-1 alpha gene and its 
interaction with hepatitis B virus mutations with hepatocellular carcinoma risk. Front Med 2014;8:217-226. doi:10.1007/s11684-014-0326-2

29. Mitchell PS, Parkin RK, Kroh EM, et al. Circulating microRNAs as stable blood-based markers for cancer detection. Proc Natl Acad Sci U S A 2008;105:10513-10518. doi:10.1073/pnas.0804549105

30. Laterza OF, Scott MG, Garrett-Engele PW, Korenblat KM, Lockwood CM. Circulating miR-122 as a potential biomarker of liver disease. Biomark Med 2013;7:205-210. doi:10.2217/bmm.12.107

31. Gehrau RC1, Mas VR, Maluf DG. Hepatic disease biomarkers and liver transplantation: what is the potential utility of microRNAs? Expert Rev Gastroenterol Hepatol 2013;7:157-170. doi:10.1586/egh.12.71
32. Rupp C, Rössler A, Halibasic E, et al. Reduction in alkaline phosphatase is associated with longer survival in primary sclerosing cholangitis, independent of dominant stenosis. Aliment Pharmacol Ther 2014;40:1292-1301. doi:10.1111/apt.12979

33. Waidmann O, Köberle V, Brunner F, Zeuzem S, Piiper A, Kronenberger B. Serum microRNA-122 predicts survival in patients with liver cirrhosis. PLoS One 2012;7:e45652. doi:10.1371/journal. pone.0045652

34. Wu X, Wu S, Tong L, et al. miR-122 affects the viability and apoptosis of hepatocellular carcinoma cells. Scand J Gastroenterol 2009;44:13321339. doi:10.3109/00365520903215305 
Supplementary Table I. Correlation between miR-122 serum levels and laboratory parameters in a second PSC cohort.

\begin{tabular}{|l|c|c|c|}
\hline Parameter & Low miR-122 group & High miR-122 group & p-value \\
\hline Patients (n) & 9 & 21 & n.a. \\
\hline $\begin{array}{l}\text { Gender, male/female, } \mathrm{n} \\
(\%)\end{array}$ & $8 / 1$ & $14 / 7$ & 0.207 \\
\hline $\begin{array}{l}\text { Age at diagnosis [years], } \\
\text { mean, SD }\end{array}$ & $33.0 \pm 14.8$ & $27.6 \pm 8.3$ & 0.218 \\
\hline IBD, $\mathrm{n}(\%)$ & $7(77.7 \%)$ & $15(71.4 \%)$ & 0.719 \\
\hline Dominant stenosis, $\mathrm{n}(\%)$ & $6(66.6 \%)$ & $16(76.1 \%)$ & 0.589 \\
\hline $\begin{array}{l}\text { Death/Liver } \\
\text { transplantation, } \mathrm{n}(\%)\end{array}$ & $6(66.6 \%)$ & $4(19.0 \%)$ & 0.011 \\
\hline $\begin{array}{l}\text { Mayo-risk score, mean, } \\
\text { SD }\end{array}$ & $0.385 \pm 1.22$ & $0.204 \pm 1.25$ & 0.069 \\
\hline ALT (U/l), mean, SD & $103.6 \pm 100.1$ & $82.0 \pm 88.6$ & 0.251 \\
\hline AST (U/l), mean, SD & $95.5 \pm 56.4$ & $70.3 \pm 46.0$ & 0.210 \\
\hline GGT (U/l), mean, SD & $211.3 \pm 152.5$ & $303.6 \pm 317.7$ & 0.416 \\
\hline ALP (U/l), mean, SD & $344.2 \pm 172.1$ & $290.3 \pm 149.6$ & 0.395 \\
\hline $\begin{array}{l}\text { Albumin (g/dl), mean, } \\
\text { SD }\end{array}$ & $4.7 \pm 0.5$ & $4.8 \pm 2.7$ & 0.436 \\
\hline $\begin{array}{l}\text { Bilirubin (mg/dl), mean, } \\
\text { SD }\end{array}$ & $2.8 \pm 1.8$ & $2.1 \pm 1.5$ & 0.126 \\
\hline INR, mean, SD & $1.08 \pm .08$ & $1.06 \pm .08$ & 0.812 \\
\hline CRP (mg/l), mean, SD & $16.6 \pm 38.6$ & $8.6 \pm 12.3$ & 0.114 \\
\hline $\begin{array}{l}\text { Leucocytes (/nl), mean, } \\
\text { SD }\end{array}$ & $7.8 \pm 2.8$ & $7.5 \pm 2.6$ & 0.662 \\
\hline
\end{tabular}

